\title{
Minimax Polynomial Approximation
}

\author{
By Harry H. Denman
}

Abstract. Some new methods for obtaining the minimax polynomial approximation of degree $n$ to a continuous function are introduced, and applied to several simple functions. The amount of computation required is substantially reduced compared with that of previous methods.

1. Introduction. In a previous paper [1], a study of the automatic generation of "optimized" subroutines for the computation of function values was initiated (using polynomial approximations). The procedure described therein was roughly the following. The programmer indicated the function $f(x)$ to be approximated (limited in [1] to $\sin \gamma x, \cos \gamma x, \exp \gamma x, \sinh \gamma x$, and $\cosh \gamma x$ ), the interval $[a, b]$ of $x$ (assumed finite), and the maximum absolute error tolerable. For these functions, the exact coefficients of their expansions in Chebyshev polynomials are known, and were calculated until the first (nonzero) coefficient in this expansion was less than the given permissible error. The resultant polynomial was then converted into a power series in $x$ for computation.

The limitations of the above work were substantial. It was assumed that the exact coefficients in the expansion of $f(x)$ in Chebyshev polynomials were known in terms of reasonably simple functions. In addition, this polynomial approximation is not the polynomial of minimum-maximum absolute error (minimax polynomial), and it is the latter which is often the desired approximation. In this paper, the "leveling" problem will be considered, i.e., a procedure for obtaining the minimax polynomial approximation to a continuous function from a "nearby" polynomial approximation. Some methods for improving the speed and accuracy of this calculation will also be presented, and several examples given.

2. Method. It is well known [2] that there exists a minimax polynomial approximation to a continuous function $f(x)$ on the closed interval $[a, b]$. Also, there exists a set of at least $n+2$ points $\left\{x_{j}^{*}\right\}$, and a minimum-maximum error $E^{*}$, such that

$$
f\left(x_{j}^{*}\right)=P_{n}^{*}\left(x_{j}^{*}\right)=(-1)^{j} E^{*},
$$

where $P_{n}^{*}(x)=\sum^{n} c_{k}{ }^{*} x^{k}$ is the minimax polynomial approximation. Let $P_{n}=$ $\sum c_{k} x^{k}$ be a polynomial of degree $n$ in $x$. Then if the set of points $\left\{x_{j, i}\right\}$ approximating $\left\{x_{j}{ }^{*}\right\}$ is used in (2.1), it can be written

$$
P_{n}\left(x_{j, i}\right)+(-1)^{j} E_{i}=f\left(x_{j, i}\right),
$$

which in general has the solution $\left(c_{k, i}, E_{i}\right)$. The error function $\epsilon_{i}(x)$ resulting from the use of $P_{n, i}(x)=\sum c_{k, i} x^{k}$ as an approximation for $f(x)$ is given by

$$
\epsilon_{i}(x)=f(x)-P_{n, i}(x) .
$$

A number of different iterative procedures for obtaining $P_{n}{ }^{*}(x)$, based on the work of Novodvorskir and Pinsker [3], have been proposed. In these procedures one

Received July 29, 1965. 
chooses an initial set of $n+2$ points $\left\{x_{j, 0}\right\}$ and substitutes them in (2.2). The solution of this set of linear equations yields the coefficients $c_{k, 0}$ and $E_{0}$ (assumed $\neq 0$ ). With these coefficients, the error function $\epsilon_{0}$ is obtained, using (2.3). Shenitzer [4] then finds the point at which $\epsilon_{0}$ attains maximum amplitude, replaces one of the $\left\{x_{j, 0}\right\}$ with it such that $\epsilon_{0}$ alternates in sign on this new set, and repeats the process until $E_{i}$ converges. (His applications were to functions defined on a point set, but the method is essentially unchanged.) The deficiencies of this method are the amount of searching required to find the maximum of $\left|\epsilon_{i}\right|$, and the fact that the equations (2.2) must be solved on each iteration and a substantial number of iterations may be required. Also, no method of choosing the initial set of points is indicated.

Fraser and Hart [5] start with the set $\left\{x_{j, 0}\right\}$ which corresponds to the extrema of $T_{n+1}(x)$, the Chebyshev polynomial of the first kind of degree $n+1$. Only the interval $[-1,1]$ was considered, as $[a, b]$ can be mapped onto $[-1,1]$. To obtain a new set of points for use in (2.2), a search is made for all the extrema of $\epsilon_{0}$ by searching over a number of steps from each $x_{j, 0}$ in the direction of increasing $\left|\epsilon_{0}\right|$ until the extrema are found approximately, then repeating the process with the new set $\left\{x_{j, 1}\right\}$, etc.

Murnaghan and Wrench [6] use the Chebyshev polynomial expansion for $f(x)$ as the first approximation to $P_{n}{ }^{*}$, i.e.,

$$
P_{n, 0}(x)=\sum_{0}^{n} a_{m} T_{m}(x) .
$$

Improvement to this approximation is made by replacing $x_{j, i}$ with the $x_{j, i+1}$ of (2.5a) below. As pointed out in [1], the Chebyshev expansion for $f(x)$ is usually not known, and, as will be shown later, a better $P_{n, 0}$ can sometimes be obtained.

In the present method, the extrema of $T_{n+1}(x)$ are used to obtain the starting set $\left\{x_{j, 0}\right\}=\{\cos j \pi /(n+1)\}, j=0,1, \cdots n+1$. (If $a_{n+1}=0$, the next Chebyshev polynomial whose coefficient $a_{m}$ is nonzero is used.) The method given below may be used to obtain succeeding sets. Also, a procedure for determining directly the changes in the coefficients of $P_{n, i}(x)$ is given. In the next section, the problem is broken into smaller parts for easier calculation of $c_{k, 0}$.

Consider the error function at the $i$ th step

$$
\epsilon_{i}(x)=f(x)-\sum c_{k, i} x^{k},
$$

where the $c_{k, i}$ have been obtained as the solution of (2.2) with the set $\left\{x_{j, i}\right\}$. To obtain a set of extrema of $\epsilon_{i}$, parabolas are passed through the points $\epsilon_{i}\left(x_{j, i}\right)$ using the expansion

$$
\epsilon_{i}(x) \doteq \epsilon_{i}\left(x_{j, i}\right)+\epsilon_{i}{ }^{\prime}\left(x_{j, i}\right)\left(x-x_{j, i}\right)+\frac{1}{2} \epsilon_{i}{ }^{\prime \prime}\left(x_{j, i}\right)\left(x-x_{j, i}\right)^{2} .
$$

To this approximation, the point $x_{j, i+1}$ where $\epsilon_{i}$ has an extremum is given by

$$
x_{j, i+1}=x_{j, i}-\epsilon_{i}^{\prime}\left(x_{j, i}\right) / \epsilon_{i}^{\prime \prime}\left(x_{j, i}\right),
$$

or

$$
\Delta x_{j, i}=x_{j, i+1}-x_{j, i}=-\epsilon_{i}^{\prime}\left(x_{j, i}\right) / \epsilon_{i}^{\prime \prime}\left(x_{j, i}\right),
$$


which is also used in [6]. In this way a new set $\left\{x_{j, i+1}\right\}$ may be generated. To insure convergence, one may test $\epsilon_{i}(x)$ to verify that it alternates on the set $\left\{x_{j, i+1}\right\}$.

In general, $\epsilon^{\prime}$ will not be 0 at the end points $a, b$, or $-1,1$. However, it is common that the end points are members of the set $\left\{x_{j}{ }^{*}\right\}$. In the examples considered here, this was the case, and therefore -1 and 1 were retained in all the sets $\left\{x_{j, i}\right\}$. Differentiating (2.4),

$$
\begin{aligned}
& \epsilon_{i}^{\prime}(x)=f^{\prime}(x)-\sum k c_{k, i} x^{k-1}, \\
& \epsilon^{\prime \prime}(x)=f^{\prime \prime}(x)-\sum k(k-1) c_{k, i} x^{k-2},
\end{aligned}
$$

so that $f(x)$ must be twice differentiable at the points $x_{j, i}$ (except the end points, which here are assumed always in the set).

Consider the changes in the set of equations (2.2) when the set $\left\{x_{j, i}\right\}$ is changed. Since the changes in the coefficients $c_{k, i}$ and the error $E_{i}$ are usually small even for appreciable changes $\Delta x_{j, i}$, only first-order terms in $\Delta c_{k, i}$ and $\Delta E_{i}$ will be retained, and terms to and including $\left(\Delta x_{j, i}\right)^{2}$. To these orders

$$
\begin{aligned}
\sum \Delta c_{k, i} x_{j, i}^{k}+(-1)^{j} \Delta E_{i}= & {\left[f^{\prime}\left(x_{j, i}\right)-\sum c_{k, i} k x_{j, i}^{k-1}\right] \Delta x_{j, i} } \\
& +\left[f^{\prime \prime}\left(x_{j, i}\right)-\sum c_{k, i} k(k-1) x_{j, i}^{k-2}\right]\left(\Delta x_{j, i}\right)^{2} / 2 .
\end{aligned}
$$

But from (2.6) and (2.7), the bracketed quantities are $\epsilon_{i}{ }^{\prime}\left(x_{j, i}\right)$ and $\epsilon_{i}{ }^{\prime \prime}\left(x_{j, i}\right)$, and (2.8) may be rewritten

$$
\sum \Delta c_{k, i} x_{j, i}^{k}+(-1)^{j} \Delta E_{i}=\epsilon^{\prime}\left(x_{j, i}\right) \Delta x_{j, i}+\epsilon^{\prime \prime}\left(x_{j, i}\right)\left(\Delta x_{j, i}\right)^{2} / 2 .
$$

If $(2.5 \mathrm{~b})$ is used to obtain $\Delta x_{j, i},(2.9)$ becomes

$$
\sum \Delta c_{k, i} x_{j, i}^{k}+(-1)^{j} \Delta E_{i}=-\left[\epsilon^{\prime}\left(x_{j, i}\right)\right]^{2} /\left[2 \epsilon^{\prime \prime}\left(x_{j, i}\right)\right] .
$$

The right side of $(2.10)$ can also be written $-\epsilon^{\prime \prime}\left(x_{j, i}\right)\left(\Delta x_{j, i}\right)^{2} / 2$, so that the changes $\Delta c_{k, i}, \Delta E_{i}$ are quadratic in the $\Delta x_{j, i}$. Unless the matrix $\left[x_{j, i}^{k},(-1)^{j}\right]$ is ill-conditioned (see Section 6 ) or $\epsilon^{\prime \prime}\left(x_{j, i}\right)$ is large, small changes in $\left\{x_{j, i}\right\}$ yield very small changes in $\left(c_{k, i}, E_{i}\right)$. Also, the coefficient matrices for $\left(c_{k, i}, E_{i}\right)$ and $\left(\Delta c_{k, i}, \Delta E_{i}\right)$ are identical.

3. Decomposition into Even and Odd Functions. From Section 2, the initial set of approximate extremal points is here chosen at the extrema of $T_{n+1}(x)$. Thus, if $x_{j, 0}$ is a member of the set, so is $-x_{j, 0}$. This permits the decomposition of $P_{n, 0}$ and $f(x)$ into even and odd parts as follows.

Case I. $n$ even.

If $n$ is even, (2.2) becomes

$$
P_{n}\left(x_{j, 0}\right)+(-1)^{j} E_{0}=f\left(x_{j, 0}\right)
$$

and for $-x_{j, 0}$,

$$
P_{n}\left(-x_{j, 0}\right)+(-1)^{j+1} E_{0}=f\left(-x_{j, 0}\right) .
$$

Adding, one obtains

$$
P_{n}^{e}\left(x_{j, 0}\right)=f^{e}\left(x_{j, 0}\right),
$$


where

$$
f^{e}(x)=[f(x)+f(-x)] / 2,
$$

and

$$
P_{n}^{e}(x)=\sum_{i=0}^{r} c_{2 i} x^{2 i}
$$

where $r=n / 2$. Only the $r+1$ points $x_{j, 0}>0$ are needed.

Subtracting (3.1b) from (3.1a), one obtains

$$
P_{n}^{o}\left(x_{j, 0}\right)+(-1)^{j} E_{0}=f^{o}\left(x_{j, 0}\right),
$$

where

$$
f^{o}(x)=[f(x)-f(-x)] / 2,
$$

and

$$
P_{n}^{o}(x)=\sum_{i=0}^{r-1} c_{2 i+1} x^{2 i+1}
$$

Again, only the $r+1$ points $x_{j, 0}>0$ are needed. If $f(x)$ is even, one can show that $P_{n}{ }^{*}(x)$ is even, and the above is not used; since $a_{n+1}=0$, the extrema of the error function are assumed approximately at the extrema of $T_{n+2}(x)$. Then (3.8) below is used at $x_{j, 0}=\cos j \pi /(n+2), j=0,1, \cdots r+1$.

Case II. $n$ odd. Similarly, for $n$ odd, one obtains

$$
P_{n}^{e}\left(x_{j, 0}\right)+(-1)^{j} E_{0}=f^{e}\left(x_{j, 0}\right),
$$

and

$$
P_{n}^{o}\left(x_{j, 0}\right)=f^{o}\left(x_{j, 0}\right) \text {, }
$$

using the $(n+3) / 2$ points $x_{j, 0} \geqq 0$. If $f(x)$ is odd, $P_{n}{ }^{*}(x)$ is odd, and (3.5) is used at $x_{j, 0}=\cos j \pi /(n+2), j=0,1, \cdots(n+1) / 2$.

Thus, if $f$ is neither even nor odd, both cases produce two smaller independent sets of simultaneous equations for $\left(c_{k, 0}, E_{0}\right)$, which substantially reduces the labor in solving them. Also, one may calculate and store the inverses of the above matrices once (with extra precision, if necessary) for reasonable $n$ values, instead of calculating them each time they are needed.

\section{Applications.}

A. The first example is a trivial one, but interesting in that, in a number of cases, it gives the exact result without any iterations. Consider any continuous even function $f^{e}$ on $[-1,1]$, for which the minimax linear polynomial approximation $c_{0}{ }^{*}+c_{1}{ }^{*} x$ is desired. Since $f^{e}$ is even, $c_{1}{ }^{*}=0$, and if $(3.8)$ is used at $\left\{x_{j, 0}\right\}=\{1,0\}$,

$$
\begin{aligned}
& c_{0}-E_{0}=f^{e}(1), \\
& c_{0}+E_{0}=f^{e}(0),
\end{aligned}
$$

or

$$
\begin{aligned}
c_{0} & =\left[f^{e}(0)+f^{e}(1)\right] / 2, \\
E_{0} & =\left[f^{e}(0)-f^{e}(1)\right] / 2 .
\end{aligned}
$$


TABLE 1

$$
f(x)=\cos (\pi x / 2) \text { in }[-1,1]
$$

\begin{tabular}{c|r|r|r|r}
\hline$k$ & \multicolumn{1}{c|}{$c_{k, 0}$} & \multicolumn{1}{c}{$c_{k, 1}$} & \multicolumn{1}{c}{$c_{k}{ }^{*}$} & \multicolumn{1}{c}{$\bar{c}_{k}$} \\
\hline 0 & 0.99940330 & 0.99940322 & 0.9994032 & 0.99939655 \\
2 & $\begin{array}{r}-1.22279688 \\
0.22399027\end{array}$ & -1.22279674 & -1.2227967 & -1.22274315 \\
4 & $E_{0}$ & $E_{1}$ & $E^{*}$ & \multicolumn{1}{c}{$\bar{E}$} \\
\hline & 0.00059670 & 0.00059677 & 0.0005968 & 0.00060345 \\
\hline
\end{tabular}

These are the minimax coefficient $c_{0}{ }^{*}$ and error $E^{*}$ if $f^{e}(0)$ and $f^{e}(1)$ are extrema of $f^{e}$ on $[-1,1]$. Such common functions as $\cos (\pi x / 2)$ and $J_{0}(x)$ satisfy this condition.

B. The second example is $f(x)=\cos (\pi x / 2)$ on $[-1,1] ; P_{5}{ }^{*}(x)$ is desired. Since $P_{5}{ }^{*}$ must be even,

$$
c_{1}=c_{3}=c_{5}=0 .
$$

Using (3.8) at $\left\{x_{j, 0}\right\}=\left\{1, \frac{1}{2} \sqrt{3}, \frac{1}{2}, 0\right\}$ one obtains

$$
\begin{aligned}
c_{0,0}+c_{2,0}+c_{4,0}-E_{0} & =0 \\
c_{0,0}+\left(\frac{3}{4}\right) c_{2,0}+\left(\frac{9}{16}\right) c_{4,0}+E_{0} & =\cos (\sqrt{3} \pi / 4) \\
c_{0,0}+\left(\frac{1}{4}\right) c_{2,0}+\left(\frac{1}{16}\right) c_{4,0}-E_{0} & =\cos (\pi / 4) \\
c_{0,0}+E_{0} & =1 .
\end{aligned}
$$

The exact inverse of the matrix $A$ above is

$$
A^{-1}=\left(\frac{1}{6}\right)\left[\begin{array}{rrrr}
1 & -2 & 2 & 5 \\
-12 & 20 & 12 & -20 \\
16 & -16 & -16 & 16 \\
-1 & 2 & -2 & 1
\end{array}\right] \text {, }
$$

and the solution of equations (4.3) is given in the first column of Table 1.

Using (2.10), the change in the coefficients $c_{k, 0}$ and $E_{0}$ may be written

$$
A \Delta_{0}=-\Gamma_{0},
$$

where $\Delta_{0}$ and $\Gamma_{0}$ are the column vectors

$$
\Delta_{0}=\left[\begin{array}{c}
\Delta c_{0,0} \\
\Delta c_{2,0} \\
\Delta c_{4,0} \\
\Delta E_{0}
\end{array}\right], \quad \Gamma_{0}=\left[\begin{array}{c}
0 \\
\left(\epsilon_{1}^{\prime}\right)^{2} / \epsilon_{1}^{\prime \prime} \\
\left(\epsilon_{2}^{\prime}\right)^{2} / \epsilon_{2}^{\prime \prime} \\
0
\end{array}\right],
$$

where $\left(\epsilon_{1}{ }^{\prime}\right)^{2} / 2 \epsilon_{1}{ }^{\prime \prime}$ is evaluated at $x_{1,0}=\sqrt{3} / 2$, and $\left(\epsilon_{2}{ }^{\prime}\right)^{2} / 2 \epsilon_{2}{ }^{\prime \prime}$ is evaluated at $x_{2,0}=\frac{1}{2}$. $\Gamma_{0,1}$ is 0 because the point $x_{0}=1$ is in the set $\left\{x_{j}{ }^{*}\right\}$, and $\Gamma_{0,4}$ is 0 because, since $\cos (\pi x / 2)$ is even, $\epsilon^{\prime}$ is 0 at $x_{4,0}$ (thus 0 is retained in the set $\left\{x_{j, i}\right\}$ also). Then

$$
\Delta_{0}=-A^{-1} \Gamma_{0}
$$


and, using (4.4), one obtains

$$
\Delta_{0}=10^{-7}\left[\begin{array}{c}
-0.75 \\
1.45 \\
0.05 \\
0.75
\end{array}\right] .
$$

When these small corrections are added to $\left(c_{k, 0}, E_{0}\right)$, the results given in the second column of Table 1 are obtained. The values $c_{k}{ }^{*}, E_{k}{ }^{*}$ are taken from [6], and agree with these results to seven decimal places. The coefficients $\bar{c}_{k}$ are those resulting from the Chebyshev expansion of $\cos (\pi x / 2) ; \bar{E}$ is their error at $x=0$.

C. The third example is $f(x)=e^{x}$ on $[-1,1] ; P_{5}{ }^{*}(x)$ is desired. (Although the interval $[-1,1]$ is used here for convenience, it is not the most appropriate interval for floating-point computations, as discussed in [1].) Then $f^{e}(x)=\cosh x$, and $f^{o}(x)=\sinh x$, and, since $n$ is odd, the set $\left\{x_{j, 0}\right\}=\left\{1, \sqrt{3} / 2, \frac{1}{2}, 0\right\}$ is used. Then (3.8) becomes

$$
A C_{0}^{e}=F_{o}^{e}
$$

where $A$ is the matrix of equations (4.3) and $C_{0}{ }^{e}$ and $F_{0}{ }^{e}$ are the column vectors

$$
C_{0}{ }^{e}=\left[\begin{array}{c}
c_{0,0} \\
c_{2,0} \\
c_{4,0} \\
E_{0}
\end{array}\right], \quad F_{0}{ }^{e}=\left[\begin{array}{c}
\cosh 1 \\
\cosh (\sqrt{3} / 2) \\
\cosh \frac{1}{2} \\
\cosh 0
\end{array}\right] .
$$

(Note that these are also the initial coefficients if $P_{5}{ }^{*}$ is desired for $\cosh x$ in $[-1,1]$.) For the odd coefficients, (3.9) yields

$$
\begin{aligned}
c_{1}+c_{3}+c_{5} & =\sinh 1, \\
c_{1}+\frac{3}{4} c_{3}+\frac{9}{16} c_{5} & =(2 / \sqrt{3}) \sinh (\sqrt{3} / 2), \\
c_{1}+\frac{1}{4} c_{3}+\frac{1}{16} c_{5} & =2 \sinh \frac{1}{2} .
\end{aligned}
$$

The matrix $B$ corresponding to these equations has the exact inverse

$$
B^{-1}=\left(\frac{1}{3}\right)\left[\begin{array}{ccc}
3 & -6 & 6 \\
-16 & 30 & -14 \\
16 & -24 & 8
\end{array}\right] \text {. }
$$

When the matrices $A^{-1}$ and $B^{-1}$ are used with the corresponding column vectors, the results $c_{k, 0}, E_{0}$ given in the first column of Table 2 are obtained.

The changes $\Delta_{0}^{e}$ (in the even coefficients and $E_{0}$ ) are given by

$$
\Delta_{0}^{e}=-A^{-1} \Gamma_{0}^{e},
$$

where

$$
\Gamma_{0}{ }^{e}=\left(\frac{1}{4}\right)\left[\begin{array}{c}
0 \\
\left(\epsilon_{1}^{\prime}\right)^{2} / \epsilon_{1}^{\prime \prime}+\left(\epsilon_{5}^{\prime}\right)^{2} / \epsilon_{5}^{\prime \prime} \\
\left(\epsilon_{2}^{\prime}\right)^{2} / \epsilon_{2}{ }^{\prime \prime}+\left(\epsilon_{4}^{\prime}\right)^{2} / \epsilon_{4}^{\prime \prime} \\
2\left(\epsilon_{3}^{\prime}\right)^{2} / \epsilon_{3}^{\prime \prime}
\end{array}\right],
$$


TABLE 2

\begin{tabular}{c|c|c|c|c}
\multicolumn{5}{|c}{$f(x)=e^{x}$ in $[-1,1]$} \\
\hline$k$ & $c_{k, 0}$ & $c_{k, 1}$ & $c_{k}{ }^{*}$ & $\bar{c}_{k}$ \\
\hline 0 & 1.00004498 & 1.00004475 & 1.00004475 & 1.00004478 \\
1 & 1.00003825 & 1.00003845 & 1.00003835 & 1.00002229 \\
2 & 0.49919516 & 0.49919697 & 0.49919699 & 0.49919676 \\
3 & 0.16642495 & 0.16642433 & 0.16642465 & 0.16648887 \\
4 & 0.04379552 & 0.04379370 & 0.04379370 & 0.04379392 \\
5 & 0.00873800 & 0.00873841 & 0.00873819 & 0.00868682 \\
\cline { 2 - 3 } & $E_{k, 0}$ & $E_{k, 1}$ & $E^{*}$ & $\bar{E}$ \\
\hline & -0.00004498 & -0.00004521 & -0.00004519 & -0.00004478 \\
\hline
\end{tabular}

where the subscripts of $\epsilon^{\prime}$ and $\epsilon^{\prime \prime}$ indicate evaluation at the points $x_{0}, x_{1}, \cdots=1$, $\sqrt{3} / 2, \frac{1}{2}, 0,-\frac{1}{2},-\sqrt{3} / 2,-1$, respectively. The changes $\Delta_{0}^{\circ}$ in the odd coefficients are given by

$$
\Delta_{0}^{0}=-B^{-1} \Gamma_{0}^{0},
$$

where

$$
\Gamma_{0}^{o}=\frac{1}{2}\left[\begin{array}{c}
0 \\
{\left[\left(\epsilon_{1}^{\prime}\right)^{2} / \epsilon_{1}^{\prime \prime}-\left(\epsilon_{5}^{\prime}\right)^{2} / \epsilon_{5}^{\prime \prime}\right] / \sqrt{3}} \\
\left(\epsilon_{2}^{\prime}\right)^{2} / \epsilon_{2}^{\prime \prime}-\left(\epsilon_{4}^{\prime}\right)^{2} / \epsilon_{4}^{\prime \prime}
\end{array}\right] .
$$

These changes result in the coefficients $c_{k, 1}$ and $E_{1}$ given in the second column of Table 2 . The even coefficients are substantially improved, while the odd coefficients are slightly over-corrected. The coefficients $c_{k}{ }^{*}$, given in the third column of Table 2, were obtained by four iterations of a procedure similar to that in [6]. Considering the size of $E^{*}$, no further iterations are indicated. The coefficients $\bar{c}_{k}$ resulting from the expansion of $e^{x}$ in Chebyshev polynomials are given in the fourth column of Table 2. $\bar{E}$ is the error, using this approximation, at $x=0$.

6. Discussion and Conclusions. In this paper, some new methods for obtaining the minimax polynomial approximation to a continuous function $f(x)$ on $[-1,1]$ have been introduced and applied to several simple functions. Basically, one seeks successive sets of points $\left\{x_{j, i}\right\}$ converging to the set $\left\{x_{j}^{*}\right\}$ which yields the minimax polynomial $P_{n}{ }^{*}$ and the minimax error $E^{*}$. The starting set $\left\{x_{j, 0}\right\}$ is chosen at the extrema of the next Chebyshev polynomial whose coefficient in the expansion of $f(x)$ is nonzero. Since this set is symmetrically distributed in $[-1,1]$, the initial problem can conveniently be broken into even and odd parts, as shown in Section 3 . The exact inverses of the matrices involved in the first step need be calculated only once.

The initial error curve $\epsilon_{0}$ is used to determine the quantities $\epsilon^{\prime}$ and $\epsilon^{\prime \prime}$ at the initial points (except for $x_{0,0}=1$ and $x_{0, n+1}=-1$, which are assumed in the set $x_{j}^{*}$ ). The first-order changes $\Delta c_{k, 0}$ and $\Delta E_{0}$ are then obtained, using the same matrix or matrices as before. If further iterations are required, the error curve $\epsilon_{1}$, resulting from the use of these modified coefficients $c_{k, 0}+\Delta c_{k, 0}$ is used to obtain the set $\left\{x_{j, 1}\right\}$ (which is, in general, not symmetric). In each iteration, the matrix formed from $\left[x_{j, i}^{k},(-1)^{j}\right]$ can be used twice, once to obtain $c_{k, i}, E_{i}$, and again to obtain changes in these quanti- 
ties. In the examples considered here, only the initial iteration was required to obtain the minimax polynomial to satisfactory accuracy.

For $f(x)=\cos (\pi x / 2)$, the coefficients $c_{k, 0}$ obtained from the extrema of $T_{6}$ are much closer to the $c_{k}{ }^{*}$ than those from $\sum_{0}^{5} a_{m} T_{m}(x)=\sum_{0}^{5} \bar{c}_{k} x^{k}$, the expansion of $\cos (\pi x / 2)$ in Chebyshev polynomials (see Table 1). However, for $f(x)=e^{x}$, the even coefficients of $\left\{c_{k, 0}\right\}$ are slightly poorer than those $\bar{c}_{k}$ obtained from the direct Chebyshev expansion, while the odd coefficients of $c_{k, 0}$ are very much better than those of $\left\{\bar{c}_{k}\right\}$ (Table 2). More important, the labor in obtaining the Chebyshev coefficients is saved.

The conditioning of the matrices used in this process, and the precision necessary in obtaining $\epsilon$, are discussed in [5]. Concerning the former, since small changes in the $x_{j, i}$ ordinarily change $c_{k, i}, E_{i}$ much less, the simultaneous equations (2.2) should be regarded as well-conditioned. If an accuracy (in approximating $f$ ) less than full single-prevision is adequate, no special treatment of (2.2) seems indicated. Assuming the required $f$ values are available, single-precision inversion appears sufficient. However, if one desires an approximation with maximum errors of a few in the last single-precision figure, greater care must be used. In such cases, this work suggests the following approach. $F_{0}, A^{-1}$, and $B^{-1}$ should be obtained with greater than single precision (although full double precision is in general greater than necessary). The matrix-vector multiplications should also be carried out in greater than single-precision. [The variable word-length of certain computers would be advantageous in this case.] Then the coefficients $c_{k, 0}$ can be obtained essentially correct to single-precision accuracy. Since the $\Delta c_{k, 0}$ are usually small, single-precision calculations would probably be adequate for their computation. Although a number of figures are lost, especially in computing $\epsilon^{\prime}$, only a few significant figures are needed for the $\Delta c_{k, 0}$. If further iterations are required, greater than single-precision would be needed for the matrix inversion and to evaluate $f\left(x_{j, i}\right)$, and again, single-precision would probably be adequate for the corrections.

In solving (2.2). Fraser and Hart replace the basis $\left\{x^{k}\right\}$ by $T_{k}(x)$ to improve conditioning. This method does not appear helpful, as the coefficients in the Chebyshev expansion decrease more rapidly than those of the power series, so that one expects more cancellation in their computation. These small coefficients are then multiplied by certain large constants (or linear combinations taken) to obtain the series coefficients $c_{k}$. (Of course, one can use an algorithm for calculating $f$ directly from the Chebyshev coefficients, but such methods ordinarily require more computation time than evaluation of the power series.)

If $f(x)$ is not differentiable, or if it is not convenient to evaluate the exact derivatives, one could instead obtain $\epsilon^{\prime}$ and $\epsilon^{\prime \prime}$ numerically. This can be done by passing a parabola through $\epsilon\left(x_{j, i}\right)$ and two nearby points to obtain the new set $\left\{x_{j, i+1}\right\}$.

In summary, it appears that these techniques can be incorporated into a generalpurpose program, which, given $n$ and some method for obtaining $f(x)$ to needed accuracy, can automatically and efficiently obtain $P_{n}{ }^{*}$.

Acknowledgment. The author wishes to thank the Computing Center at Wayne State University for making computer time available for a portion of this work, and Professor C. Briggs, with whom the author had a number of stimulating discussions. 
1. Harry H. Denman, "Computer generation of optimized subroutines," J. Assoc. Comput. Mach., v. 8, 1961, pp. 104-116.

2. N. I. Achieser, Theory of Approximation, (English translation), Ungar, New York, 1956, p. 57. MR 20 \#1872.

3. E. P. Novordovskil \& I S. Pinsker, "The process of equating maxima," Uspehi Mat. $N a u k$, v. 6, 1951, no. 6 (46), pp. 174-181. MR 13, 728.

4. A. Shenitzer, "Chebyshev approximation of a continuous function by a class of functions," J. Assoc. Comput. Mach., v. 4, 1957, pp. 30-35.

5. W. Fraser \& J. F. Hart, "On the computation of rational approximations to continuous functions," Comm. ACM, v. 5, 1962, pp. 401-403.

6. F. D. Murnaghan \& J. W. Wrench, "The determination of the Chebyshev approximating polynomial for a differentiable function," $M T A C, \mathrm{v} .13,1959$, pp. 185-193. MR 21 \#4526. 